International Journal of Pure and Applied Mathematics

Volume 86 No. 2 2013, 425-442

ISSN: 1311-8080 (printed version); ISSN: 1314-3395 (on-line version)

url: http://www.ijpam.eu

doi: http://dx.doi.org/10.12732/ijpam.v86i2.16

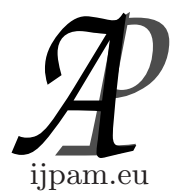

\title{
ON THE NUMERICAL SOLUTIONS OF THREE-DIMENSIONAL MHD STAGNATION-POINT FLOW OF A NEWTONIAN FLUID
}

\author{
Alessandra Borrelli ${ }^{1} \S$, Giulia Giantesio ${ }^{2}$, Maria Cristina Patria ${ }^{3}$ \\ 1,2,3 Department of Mathematics and Computer Science \\ via Machiavelli, 35, Ferrara, ITALY
}

\begin{abstract}
In this paper the steady three-dimensional stagnation-point flow of an incompressible, homogeneous, electrically conducting Newtonian fluid over a flat plate is investigated numerically. The fluid is permeated by a uniform external magnetic field $\mathbf{H}_{0}$. The effects of the magnetic field on the velocity profiles are presented graphically and discussed. This paper completes the analysis concerning the Newtonian fluids devoleped in [4].

The obtained results indicate that the thickness of the boundary layer decreases when the magnetic field increases. Moreover $\mathbf{H}_{0}$ tends to prevent the occurrence of the reverse flow.

By virtue of the numerical integration, the stagnation-point is classified as nodal or saddle point and as attachment or separation point.
\end{abstract}

AMS Subject Classification: 76W05, 76D10

Key Words: Newtonian fluids, MHD flow, three-dimensional stagnationpoint flow, numerical solutions

Received: April 10, 2013

(C) 2013 Academic Publications, Ltd. url: www.acadpubl.eu

${ }^{\S}$ Correspondence author 


\section{Introduction}

The three-dimensional stagnation-point flow occurs when a jet of fluid impinges on a rigid body. This is an important example of flow where the three velocity components appear.

This motion has been object of many investigations starting from the paper of Homman in 1936 ([1], [2], [3]). Through similarity transformations, the study of such a flow is reduced to a non-linear ordinary differential boundary value problem. The obtained system depends upon a parameter $c>-1$ which is a measure of three-dimensionality.

The aim of this paper is to complete our previous analysis concerning the steady three-dimensional flow of an incompressible, homogeneous, electrically conducting Newtonian fluid permeated by a uniform external magnetic field $\mathbf{H}_{0}$ near a three-dimensional stagnation-point of a rigid wall ([4]). The coordinate axes are fixed so that the stagnation-point is the origin and the rigid wall coincides with the plane $x_{2}=0$.

In [4] we have proved that, if we impress an external magnetic field $\mathbf{H}_{0}$, and we neglect the induced magnetic field (as it is customary when the magnetic Reynolds number is very small), then the steady three-dimensional MHD stagnation-point flow is possible if, and only if, $\mathbf{H}_{0}$ has the direction of one of the coordinate axes. In all cases by means of similarity transformations, we find that the flow has to satisfy a non-linear ordinary differential boundary value problem whose form depends on the direction of $\mathbf{H}_{0}$. The magnetic field influences the solutions of the three problems through the Hartmann number $M^{2}$.

In this paper we solve the boundary value problems by numerical integration, because they are not solvable by means of analytical functions due to non-linearity.

We underline that if $M^{2}=0$, then the systems we find reduce to the system governing the flow of a Newtonian fluid in the absence of the magnetic field. For this last problem, some results concerning existence are known in the literature ([5], [6], [3]). In the presence of the magnetic field, there are not theoretical results concerning the existence of solutions. Moreover from the numerical point of view we underline that the case in which $\mathbf{H}_{0}$ is orthogonal to the rigid wall (our Case II) is the unique one usually studied in the literature.

By means of our numerical results, we find that $\mathbf{H}_{0}$ tends to prevent the reverse flow which occurs in the absence of the magnetic field for suitable negative values of $c([3])$. The influence of the magnetic field on the reverse flow was also found in [7] in other physical situations. 
The presence of $\mathbf{H}_{0}$ modifies also the thickness of the boundary layer which decreases as $M^{2}$ increases. This effect occurs in all cases studied and it is standard in MHD.

By using numerical integration, we classify the stagnation-point as nodal or saddle point and as attachment or separation point. The classification depends on $c$ and $M^{2}$. In Case II and Case III, as for $M^{2}=0$, the origin is a point of attachment. In Case I, we find a new result: when $M^{2}$ is sufficiently large and $c$ assumes suitable negative values, then the stagnation-point becomes a separation point. Further in all the three cases, if $c>0$ or where there is the reverse flow, the origin is a nodal point, while when $c<0$ and the reverse flow does not appear, it is a saddle point.

We notice that from a theoretical point of view, MHD flows of this type are fundamental in fluid dynamics. From a practical point of view, stagnation-point flows are ubiquitous in the sense that they inevitably appear as a component of more complicated flow fields. So the investigation in this area is motivated by the possibility of solving exactly the boundary layer equations at the stagnation point and by their relevance to a wide range of engineering, industrial and technical applications. The paper is organized in this way:

In Section 2, we formulate the problem, recall the results contained in [4] and define the nodal, saddle, attachment and separation points.

In Section 3, we integrate numerically the previous problems, and discuss the behaviour of the solutions.

Section 4 is devoted to conclusions.

\section{Overview of the Problem}

In [4] we have studied the steady three-dimensional MHD stagnation-point flow of an incompressible homogeneous electrically conducting Newtonian fluid towards a flat surface. We assume that the flat surface is the rigid, fixed, nonelectrically conducting wall of equation $x_{2}=0$, so that the flow is confined to the half-space $\mathcal{S}=\left\{\left(x_{1}, x_{2}, x_{3}\right) \in \mathbb{R}^{3}:\left(x_{1}, x_{3}\right) \in \mathbb{R}^{2}, x_{2}>0\right\}$ (see Figure 1 ).

As it is well known ([8], [3]), the three-dimensional stagnation-point flow is described by a velocity field of the form

$$
v_{1}=a x_{1} f^{\prime}(\eta), \quad v_{2}=-\sqrt{\nu a}[f(\eta)+c g(\eta)], \quad v_{3}=c a x_{3} g^{\prime}(\eta),
$$

where $a$ is a positive parameter, $c$ is a constant which is a measure of threedimensionality, $f, g$ are sufficiently regular unknown dimensionless functions 


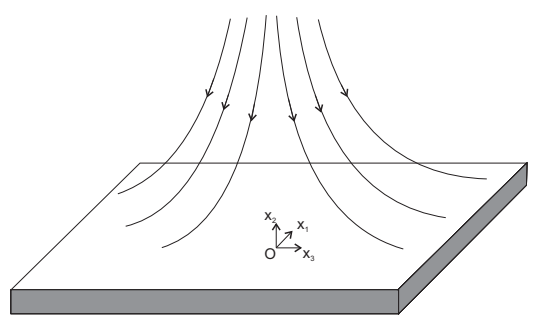

Figure 1: Flow description.

and $\eta=\sqrt{\frac{a}{\nu}} x_{2}$. We exclude in our analysis $c=0$, because this describes the orthogonal plane stagnation-point flow .

Moreover the usual adherence condition on the boundary supplies

$$
f(0)=0, \quad f^{\prime}(0)=0, \quad g(0)=0, \quad g^{\prime}(0)=0 .
$$

As it is customary, we assume that at infinity, the flow approaches the flow of an inviscid fluid impinging on the flat plane $\eta=h([3],[4])$, whose velocity is given by:

$$
v_{1}=a x_{1}, \quad v_{2}=-\sqrt{\nu a}(1+c)(\eta-h), \quad v_{3}=\operatorname{cax}_{3}, \quad \eta \geq h .
$$

The constant $h$ in (3) is the three-dimensional displacement thickness ([3]) and it is related to the behaviour of $f$ and $g$ at infinity. Actually, if

$$
\lim _{\eta \rightarrow+\infty}[f(\eta)-\eta]=-\alpha, \lim _{\eta \rightarrow+\infty}[g(\eta)-\eta]=-\beta
$$

with $\alpha, \beta$ some constants, then

$$
\lim _{\eta \rightarrow+\infty}[f(\eta)+c g(\eta)-(1+c) \eta]=-(1+c) h
$$

from which

$$
h=\frac{\alpha+c \beta}{1+c} .
$$

The constants $\alpha, \beta$ are not assigned a priori, but their values can be found by solving the problem. As we will see, the three-dimensional displacement thickness $h$ can be positive or negative.

We exclude the case $c \leq-1$ because we impose in (3) the condition $v_{2}<0$, so that the inviscid fluid moves towards the wall $\eta=h$. Hence we assume $c \in(-1,0) \cup(0,+\infty)$.

In [4] we have proved the following 
Theorem 1. Let a homogeneous, incompressible, electrically conducting Newtonian fluid occupy the half-space $\mathcal{S}$. If we impress the external magnetic field $\mathbf{H}_{0}$ parallel to one of the axes (with unit vector $\mathbf{e}_{i}$ for $i=1,2,3$ ) and if we neglect the induced magnetic field, then the steady three-dimensional MHD stagnation-point flow of such a fluid has the form (1), $\mathbf{E}=\mathbf{0}$, and

(I) if $\mathbf{H}_{0}=H_{0} \mathbf{e}_{1}$, then $(f, g)$ satisfies the problem

$$
\begin{aligned}
& f^{\prime \prime \prime}+(f+c g) f^{\prime \prime}-f^{\prime 2}+1=0, \\
& g^{\prime \prime \prime}+(f+c g) g^{\prime \prime}-c g^{2}+c+M^{2}\left(1-g^{\prime}\right)=0, \\
& f(0)=0, \quad f^{\prime}(0)=0, \quad g(0)=0, \quad g^{\prime}(0)=0, \\
& \lim _{\eta \rightarrow+\infty} f^{\prime}(\eta)=1, \quad \lim _{\eta \rightarrow+\infty} g^{\prime}(\eta)=1,
\end{aligned}
$$

where $M^{2}$ is the Hartmann number ${ }^{1}$;

(II) if $\mathbf{H}_{0}=H_{0} \mathbf{e}_{2}$, then $(f, g)$ satisfies

$$
\begin{aligned}
& f^{\prime \prime \prime}+(f+c g) f^{\prime \prime}-f^{\prime 2}+1+M^{2}\left(1-f^{\prime}\right)=0, \\
& g^{\prime \prime \prime}+(f+c g) g^{\prime \prime}-c g^{\prime 2}+c+M^{2}\left(1-g^{\prime}\right)=0,
\end{aligned}
$$

with the boundary conditions (7);

(III) if $\mathbf{H}_{0}=H_{0} \mathbf{e}_{3}$, then $(f, g)$ satisfies

$$
\begin{aligned}
& f^{\prime \prime \prime}+(f+c g) f^{\prime \prime}-f^{\prime 2}+1+M^{2}\left(1-f^{\prime}\right)=0, \\
& g^{\prime \prime \prime}+(f+c g) g^{\prime \prime}-c g^{\prime 2}+c=0,
\end{aligned}
$$

with the boundary conditions (7).

Remark 2. If $M^{2}=0$, then equations (6), (8), (9) reduce to

$$
\begin{aligned}
& f^{\prime \prime \prime}+(f+c g) f^{\prime \prime}-f^{\prime 2}+1=0, \\
& g^{\prime \prime \prime}+(f+c g) g^{\prime \prime}-c g^{2}+c=0
\end{aligned}
$$

\footnotetext{
${ }^{1}$ For the convenience of the reader, we recall that $M^{2}=\frac{\sigma_{e} \mu_{e}^{2} H_{0}^{2}}{\rho a}$, where $\sigma_{e}$ is the electrical conductivity, $\mu_{e}$ is the magnetic permeability, $\rho$ is the mass density of the fluid (positive constants).
} 
which are the dimensionless equations governing the three-dimensional stagnationpoint flow of a Newtonian fluid in the absence of $\mathbf{H}_{0}$. In [3] it is proved that the problem (10), (7) does not admit solution for $c<-1$. As far as existence of solutions is concerned, we refer to [5], [6].

Remark 3. In [4] we have shown that the pressure field which corresponds to the three problems of the previous theorem is respectively :

$$
\begin{aligned}
p= & -\rho \frac{a^{2}}{2}\left\{x_{1}^{2}+\frac{\nu}{a}[f(\eta)+c g(\eta)]^{2}+c^{2} x_{3}^{2}\right\}-\rho a \nu\left[f^{\prime}(\eta)+c g^{\prime}(\eta)\right] \\
& +\rho a^{2} M^{2}\left\{\frac{\nu}{a} \int_{0}^{\eta}[f(s)+c g(s)] d s-\frac{c}{2} x_{3}^{2}\right\}+p_{0}, \\
p= & -\rho \frac{a^{2}}{2}\left\{x_{1}^{2}+\frac{\nu}{a}[f(\eta)+c g(\eta)]^{2}+c^{2} x_{3}^{2}\right\}-\rho a \nu\left[f^{\prime}(\eta)+c g^{\prime}(\eta)\right] \\
& -\rho a^{2} M^{2}\left(x_{1}^{2}+c x_{3}^{2}\right)+p_{0}, \\
p= & -\rho \frac{a^{2}}{2}\left\{x_{1}^{2}+\frac{\nu}{a}[f(\eta)+c g(\eta)]^{2}+c^{2} x_{3}^{2}\right\}-\rho a \nu\left[f^{\prime}(\eta)+c g^{\prime}(\eta)\right] \\
& +\rho a^{2} M^{2}\left\{\frac{\nu}{a} \int_{0}^{\eta}[f(s)+c g(s)] d s-\frac{x_{1}^{2}}{2}\right\}+p_{0} .
\end{aligned}
$$

It is important to explicit the pressure field because, as it is well known, when a fluid moves past a body, if one of the components of the pressure gradient along a body surface has the same sign of the corresponding component of the velocity, then the reverse flow appears.

In the absence of the external magnetic field for the three-dimensional stagnation-point flow, the numerical results ([3], [9]) show that there exists a negative value of $c\left(c_{r}\right)$ such that if $c \geq c_{r}$, then $g^{\prime}, g^{\prime \prime}>0 \forall \eta>0$, and if $c<c_{r}$ then near the wall $g^{\prime}, g^{\prime \prime}<0$, so that the reverse flow appears (i.e. $v_{3}$ has the same sign of $\frac{\partial p}{\partial x_{3}}$ ).

The reverse flow is also related to a sign change of the scalar component of the skin friction $\left(\tau_{\mathbf{0}}\right)$ in the direction of $\mathbf{e}_{3}$ (see (15).

When an external magnetic field $\mathbf{H}_{0}$ is impressed, as one can see from (11), (12), (13), the pressure field depends on $\mathbf{H}_{0}$ through the Hartmann number $M^{2}$, which influences the sign of the components of the pressure gradient along the wall. For this reason, as we will see in the next section, the presence of the external magnetic field tends to prevent the occurrence of the reverse. This 
behaviour appears more clearly in Cases I-II, and it has also been observed in [7] in other physical situations.

Remark 4. As it is underlined in [10], at a very small distance $\eta$ from the surface of an obstacle, the velocity of a Newtonian fluid is approximately

$$
\mathbf{v} \cong \sqrt{\frac{\nu}{a}} \frac{\tau_{\mathbf{0}}}{\mu} \eta
$$

where $\tau_{\mathbf{0}}$ is the skin friction vector, which in our situation is given by

$$
\tau_{\mathbf{0}}=\rho a \sqrt{\nu a}\left[x_{1} f^{\prime \prime}(0) \mathbf{e}_{1}+c x_{3} g^{\prime \prime}(0) \mathbf{e}_{3}\right] .
$$

Moreover the normal component of the velocity at a higher order of approximation is

$$
v_{2} \cong-\frac{1}{2} \frac{\nu}{a} \operatorname{div}\left(\frac{\tau_{0}}{\mu}\right) \eta^{2}:=-\frac{1}{2} \Delta_{s} \eta^{2} .
$$

We see from (14) that close to the obstacle the direction of streamlines becomes parallel to its surface, except where $\tau_{\mathbf{0}}=\mathbf{0}$. This condition that both tangential components of skin friction vanish simultaneously, is satisfied in general only at isolated points of the surface, which are called 'points of separation' if $\Delta_{s}<0$ (so that the normal velocity (16) is positive) and 'points of attachment' if $\Delta_{s}>0$.

In our analysis, the only isolated point such that $\tau_{\mathbf{0}}=\mathbf{0}$ is the origin, i.e. the stagnation-point, and

$$
\Delta_{s}=\sqrt{\nu a}\left[f^{\prime \prime}(0)+c g^{\prime \prime}(0)\right] .
$$

Streamlines very near to the surface lie closely along the skin friction line, as (14) indicates.

There is just one skin friction line and one vortex line through each point of the surface, except a point of attachment or separation. These last are 'singular points' of the differential equations of both systems of curves. Such singular points are classified into two main types, depending on the sign of:

$$
J_{s}=\frac{\partial \tau_{01}}{\partial x_{1}} \frac{\partial \tau_{03}}{\partial x_{3}}-\frac{\partial \tau_{01}}{\partial x_{3}} \frac{\partial \tau_{03}}{\partial x_{1}} .
$$

A singular point where $J_{s}<0$ is a 'saddle point', and where $J_{s}>0$ there is a 'nodal point'.

We remark that we have

$$
J_{s}=\rho^{2} \nu a^{3}\left[c f^{\prime \prime}(0) g^{\prime \prime}(0)\right] .
$$


From these considerations it is clear that we need to know the signs of $c, f^{\prime \prime}(0)$, $g^{\prime \prime}(0)$ in order to classify the stagnation-point.

As it is underlined in [9], ${ }^{2}$ in the case $M^{2}=0$ from the numerical results one has that the stagnation-point is a nodal point of attachment if $c>0$ or $c<c_{r}=-0.4294$, while it is a saddle point of attachment if $c_{r} \leq c<0$.

Finally there is a limiting direction of our flow at the boundary, which is also the direction of the resultant skin friction, and this direction is inclined to the main stream at an angle $\epsilon$ :

$$
\epsilon=\arctan \left(c \frac{x_{3}}{x_{1}} \frac{g^{\prime \prime}(0)}{f^{\prime \prime}(0)}\right)-\arctan \left(c \frac{x_{3}}{x_{1}}\right) .
$$

\section{Numerical Results and Discussion}

In this section we discuss the numerical solutions of the three problems here considered. These numerical solutions are obtained using the MATLAB routine bvp4c described in [11].

The values of the parameter $c$ are chosen according to [3], [2].

In the sequel, we will see that the solution $(f, g)$ of the three problems here considered satisfies the conditions (4); therefore we define:

- $\bar{\eta}_{f}\left(\bar{\eta}_{g}\right)$ the value of $\eta$ such that $f^{\prime}\left(\bar{\eta}_{f}\right)=0.99\left(g^{\prime}\left(\bar{\eta}_{g}\right)=0.99\right)$.

Hence if $\eta>\bar{\eta}_{f}\left(\eta>\bar{\eta}_{g}\right)$, then $f \cong \eta-\alpha(g \cong \eta-\beta)$.

From the numerical integration we will find that the influence of the viscosity on the velocity appears only in a layer lining the boundary whose thickness is $\delta=\max \left(\bar{\eta}_{f}, \bar{\eta}_{g}\right)$.

\subsection{Case I: $\mathrm{H}_{0}=\mathrm{H}_{0} \mathrm{e}_{1}$}

We have solved problem (6), (7) numerically.

Figure 2 shows the graphics of $f, f^{\prime}, f^{\prime \prime}$ for $M^{2}=1$, and $c=0.25$. As one can see,

$$
\lim _{\eta \rightarrow+\infty} f^{\prime \prime}(\eta)=0, \quad \lim _{\eta \rightarrow+\infty} f^{\prime}(\eta)=1 .
$$

The numerical integration furnishes the value of $\alpha$ when $M^{2}$ and $c$ change, as it is shown in Table 1.

\footnotetext{
${ }^{2}$ We remark that in [9] the Author corrected the classification of the stagnation-point contained in [3]. In the literature, most of the papers refer to the uncorrected classification in [3].
} 


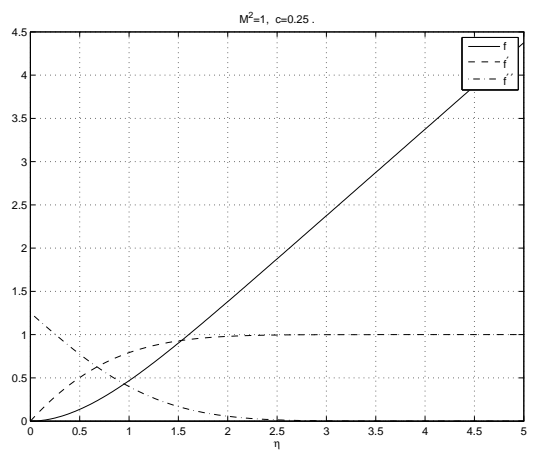

Figure 2: Case I: $f$ profile for $M^{2}=1$ and $c=0.25$.

\begin{tabular}{cccccccccc}
\hline$M^{2}$ & $c$ & $f^{\prime \prime}(0)$ & $g^{\prime \prime}(0)$ & $h$ & $\alpha$ & $\beta$ & $\bar{\eta}_{f}$ & $\bar{\eta}_{g}$ & $\delta$ \\
\hline 0 & -0.75 & 1.2465 & -0.4690 & -5.0704 & 0.6378 & 2.5405 & 2.3772 & 4.5298 & 4.5298 \\
0 & -0.25 & 1.2251 & 0.2681 & 0.4211 & 0.6593 & 1.3741 & 2.4684 & 3.6223 & 3.6223 \\
0 & 0.25 & 1.2476 & 0.8051 & 0.6699 & 0.6294 & 0.8317 & 2.2624 & 2.6363 & 2.6363 \\
0 & 1.00 & 1.3119 & 1.3119 & 0.5689 & 0.5689 & 0.5689 & 1.9444 & 1.9444 & 1.9444 \\
1 & -0.75 & 1.2051 & 0.4660 & -1.8314 & 0.6897 & 1.5300 & 2.7009 & 4.4766 & 4.4766 \\
1 & -0.25 & 1.2166 & 0.9234 & 0.6001 & 0.6692 & 0.8768 & 2.5219 & 3.0639 & 3.0639 \\
1 & 0.25 & 1.2521 & 1.2537 & 0.6300 & 0.6253 & 0.6487 & 2.2449 & 2.3264 & 2.3264 \\
1 & 1.00 & 1.3216 & 1.6383 & 0.5288 & 0.5627 & 0.4950 & 1.9227 & 1.7971 & 1.9227 \\
5 & -0.75 & 1.1543 & 1.9982 & 1.5127 & 0.7639 & 0.5143 & 3.1703 & 2.3676 & 3.1703 \\
5 & -0.25 & 1.2055 & 2.1771 & 0.7593 & 0.6812 & 0.4470 & 2.5788 & 1.9361 & 2.5788 \\
5 & 0.25 & 1.2602 & 2.3433 & 0.5752 & 0.6188 & 0.4011 & 2.2191 & 1.6772 & 2.2191 \\
5 & 1.00 & 1.3434 & 2.5735 & 0.4514 & 0.5498 & 0.3530 & 1.8797 & 1.4309 & 1.8797 \\
\hline
\end{tabular}

Table 1: Case I: descriptive quantities of motion for some values of $c$ and $M^{2}$

Our results are consistent with the previous studies when $M^{2}=0$ ([3], [2]). In particular when $M^{2}=0$ and $c=1$ we obtain the $\eta$ - axial symmetric flow: $\alpha=\beta=h$ and $f^{\prime \prime}(0)=g^{\prime \prime}(0)$. We notice that there are no results in the literature if $M^{2} \neq 0$.

As far as the behaviour of $g, g^{\prime}, g^{\prime \prime}$ is concerned, we recall that $c_{r}$ is the negative value of $c$ such that if $c \geq c_{r}$, then $g^{\prime}>0 \forall \eta>0$, and if $c<c_{r}$ then the reverse flow appears at a very small distance from the wall, i.e. $v_{3}$ has the same sign of $\frac{\partial p}{\partial x_{3}}$. If $c<c_{r}$ then the behaviour of $g, g^{\prime}, g^{\prime \prime}$ is shown in Figure $3_{1}$, otherwise it is given in Figure $3_{2}$.

Table 1 shows that if $M^{2}$ is fixed, then when $c$ increases, the values of $f^{\prime \prime}(0), g^{\prime \prime}(0)$ increase, while the values of $\alpha, \beta, \bar{\eta}_{f}, \bar{\eta}_{g}$ decrease. Hence the 

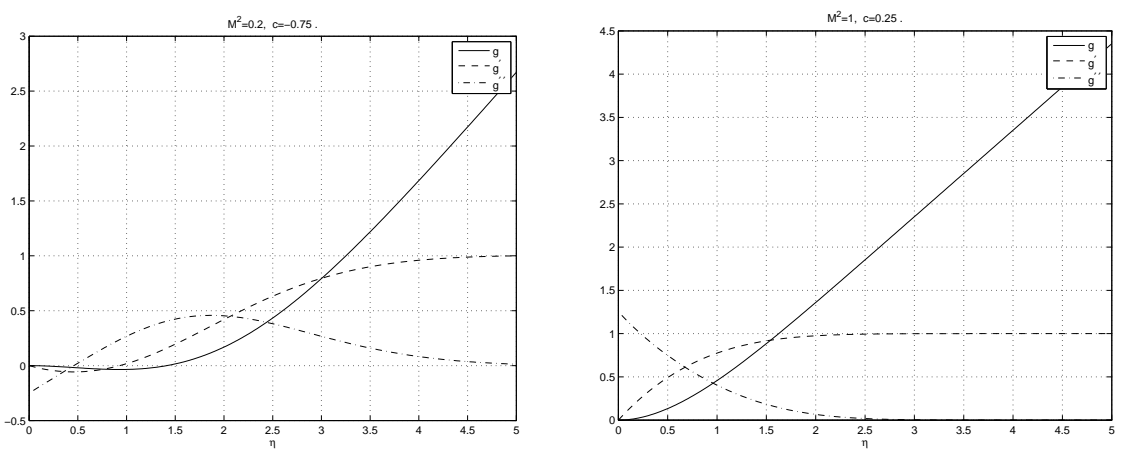

Figure 3: Case I: The first picture shows the profile of $g$ in the reverse flow $\left(M^{2}=0.2, c=-0.75, \delta=4.5482\right)$. The second picture shows the profile of $g$ in the absence of the reverse flow $\left(M^{2}=1, c=0.25\right)$.

thickness of the boundary layer decreases when $c$ increases.

In Table 1, we see also the values of the descriptive quantities of the motion when $M^{2}$ increases. In this case $g^{\prime \prime}(0)$ and $\bar{\eta}_{f}$ increase, while $\beta$ and $\bar{\eta}_{g}$ decrease. As far as $f^{\prime \prime}(0)$ and $\alpha$ are concerned, when $M^{2}$ increases, we have that $f^{\prime \prime}(0)$ increases if $c>0$, otherwise decreases, and $\alpha$ decreases if $c>0$, otherwise increases.

We have that the thickness $\delta$ of the boundary layer depends on $M^{2}$ and decreases when $M^{2}$ increases (as easily seen in Figures 4). This effect is standard in magnetohydrodynamics.

Finally from Table 1 we remark that the value of $h$, which is the the hight of the plane towards which the inviscid fluid moves, regardless of the values of $M^{2}$, increases if $c<0$, while it decreases if $c>0$.

Table 2 shows that as the Hartmann number $M^{2}$ increases, the value of $c_{r}$ for which the reverse flow does not occur (i.e. when $g^{\prime \prime}(0)=0$ ) decreases and when $M^{2}=0.8123$, the reverse flow does not occur at all for any value of $c$.

Hence the magnetic field prevents the occurrence of the reverse flow. This fact could be explained by observing that

$$
\frac{\partial p}{\partial x_{3}}=-\rho a^{2} c x_{3}\left(c+M^{2}\right)
$$

from which one can see that the signs of $c$ and of $\left(c+M^{2}\right)$ modify the sign of $\frac{\partial p}{\partial x_{3}}$.

As far as the classification of the stagnation-point is concerned, we have found a very interesting new result: for negative values of $c$ when $M^{2} \geq 2.6662$, 

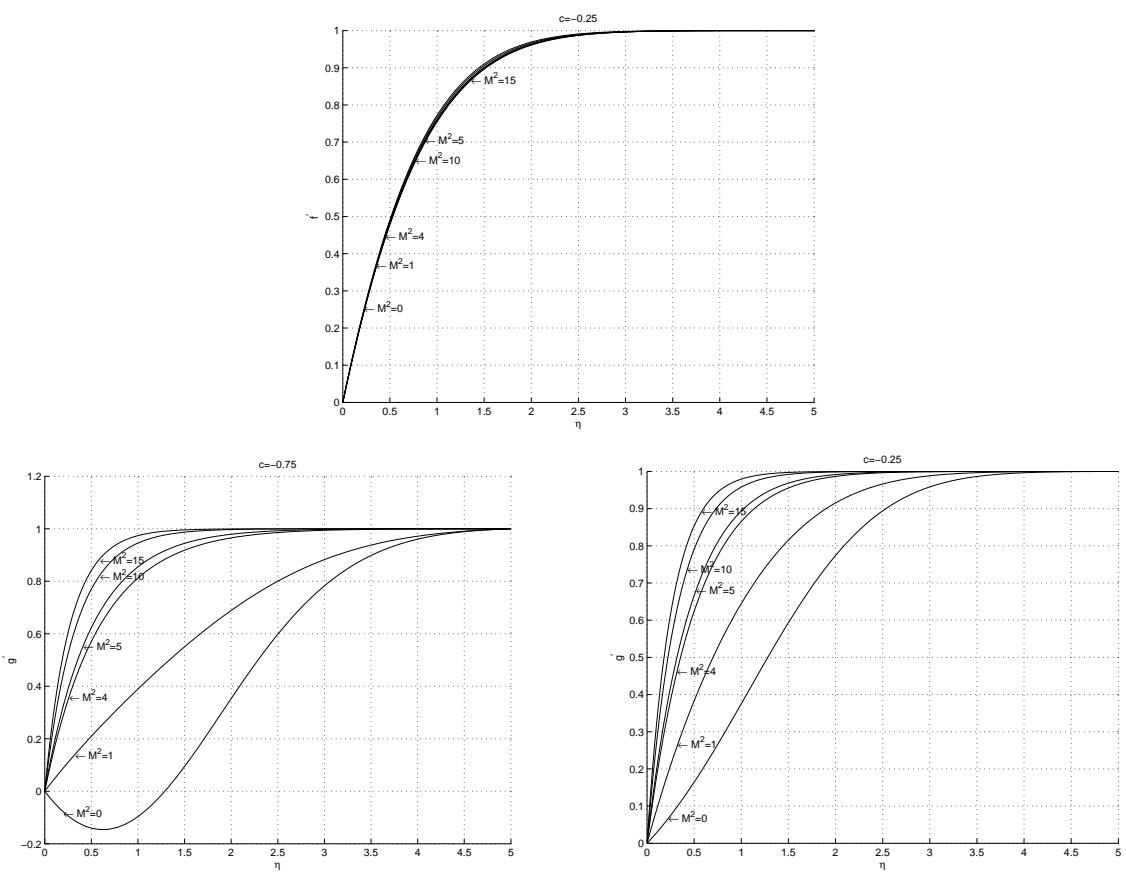

Figure 4: Case I: profiles of $f^{\prime}\left(4_{1}\right)$ and $g^{\prime}\left(4_{2,3}\right)$ for several values of $M^{2}$.

\begin{tabular}{cc}
\hline$M^{2}$ & $c_{r}$ \\
\hline 0.00 & -0.4294 \\
0.10 & -0.4991 \\
0.30 & -0.6393 \\
0.50 & -0.7802 \\
0.70 & -0.9210 \\
0.80 & -0.9913 \\
0.8122 & -0.9999 \\
0.8123 & no reverse flow \\
\hline
\end{tabular}

Table 2: Case I: Values $c_{r}$ when $M^{2}$ increases

the origin becomes a point of separation, unlike of what occurs in the absence of the magnetic field or in the next two cases, as we will see. We note that if $M^{2}<2.6662$, then the stagnation-point is always a point of attachment. Moreover if $c>0$ or where there is the reverse flow, the origin is a nodal point, while when $c<0$ and the reverse flow does not appear, it is a saddle point (as we can see from Table 3 ). 


\begin{tabular}{ccccc}
\hline$M^{2}$ & $c$ & $f^{\prime \prime}(0)+c g^{\prime \prime}(0)$ & $f^{\prime \prime}(0)$ & $g^{\prime \prime}(0)$ \\
\hline 0 & -0.90 & 1.8438 & 1.2592 & -0.6495 \\
0 & -0.75 & 1.5982 & 1.2465 & -0.4690 \\
0 & -0.50 & 1.2844 & 1.2301 & -0.1087 \\
0 & -0.25 & 1.1581 & 1.2251 & 0.2681 \\
0 & -0.10 & 1.1825 & 1.2284 & 0.4594 \\
1 & -0.90 & 0.9409 & 1.2097 & 0.2987 \\
1 & -0.75 & 0.8556 & 1.2051 & 0.4660 \\
1 & -0.50 & 0.8481 & 1.2064 & 0.7166 \\
1 & -0.25 & 0.9858 & 1.2166 & 0.9234 \\
1 & -0.10 & 1.1225 & 1.2257 & 1.0319 \\
10 & -0.90 & -1.5431 & 1.1165 & 2.9552 \\
10 & -0.75 & -1.1093 & 1.1355 & 2.9930 \\
10 & -0.50 & -0.3599 & 1.1676 & 3.0550 \\
10 & -0.25 & 0.4212 & 1.2002 & 3.1160 \\
10 & -0.10 & 0.9044 & 1.2196 & 3.1520 \\
30 & -0.90 & -3.7355 & 1.0857 & 5.3570 \\
30 & -0.75 & -2.9234 & 1.1101 & 5.3780 \\
30 & -0.50 & -1.5552 & 1.1513 & 5.4129 \\
30 & -0.25 & -0.1696 & 1.1923 & 5.4475 \\
30 & -0.10 & 0.6698 & 1.2166 & 5.4682 \\
\hline
\end{tabular}

Table 3: Case I: values of $f^{\prime \prime}(0)+c g^{\prime \prime}(0)$ for several values of $c, M^{2}$

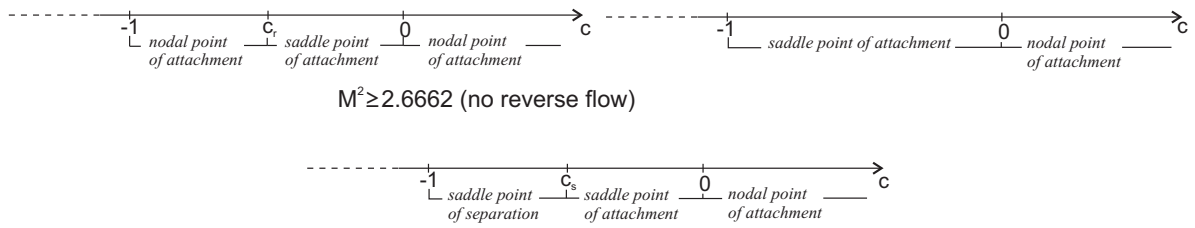

Figure 5: Case I: classification of the stagnation-point in dependence on $M^{2}$ and $c$

In Table 4, for some values of $M^{2} \geq 2.6662$, we list the negative value of $c\left(c_{s}\right)$, for which if $c<c_{s}$ then the origin is a separation point, while if $c \geq c_{s}$ then it is an attachment point. The change of the origin from attachment point to separation point could be explained by the form of system (6). Since $M^{2}$ directly influences $g$ and only indirectly influences $f$, then when $M^{2}$ increases, $g^{\prime \prime}(0)$ becomes much greater than $f^{\prime \prime}(0)$ as we can see from tables 1,2 .

In order to summarize the classification of the stagnation-point in dependence on $M^{2}$ and $c$, we provide Figure 5 . 


\begin{tabular}{cc}
\hline$M^{2}$ & $c_{s}$ \\
\hline 4.00 & -0.6593 \\
5.00 & -0.5677 \\
10.00 & -0.3836 \\
15.00 & -0.3110 \\
\hline
\end{tabular}

Table 4: Case I: Values of $c_{s}$ when $M^{2}$ increases (separation point attachment point)

\begin{tabular}{cccccccccc}
\hline$M^{2}$ & $c$ & $f^{\prime \prime}(0)$ & $g^{\prime \prime}(0)$ & $h$ & $\alpha$ & $\beta$ & $\bar{\eta}_{f}$ & $\bar{\eta}_{g}$ & $\delta$ \\
\hline 1 & -0.75 & 1.5678 & 0.4894 & -2.1123 & 0.5628 & 1.4544 & 2.3006 & 4.3855 & 4.3855 \\
1 & -0.25 & 1.5752 & 0.9395 & 0.4523 & 0.5522 & 0.8521 & 2.1961 & 2.9803 & 2.9803 \\
1 & 0.25 & 1.5980 & 1.2647 & 0.5505 & 0.5286 & 0.6385 & 2.0156 & 2.2864 & 2.2864 \\
1 & 1.00 & 1.6453 & 1.6453 & 0.4910 & 0.4910 & 0.4910 & 1.7797 & 1.7797 & 1.7797 \\
5 & -0.75 & 2.5290 & 2.0177 & 0.0298 & 0.3796 & 0.4962 & 1.6836 & 2.2264 & 2.2264 \\
5 & -0.25 & 2.5429 & 2.1942 & 0.3489 & 0.3706 & 0.4358 & 1.5941 & 1.8581 & 1.8581 \\
5 & 0.25 & 2.5589 & 2.3582 & 0.3680 & 0.3616 & 0.3935 & 1.5114 & 1.6282 & 1.6282 \\
5 & 1.00 & 2.5858 & 2.5858 & 0.3484 & 0.3484 & 0.3484 & 1.4025 & 1.4025 & 1.4025 \\
\hline
\end{tabular}

Table 5: Case II: descriptive quantities of motion for some values of $c$ and $M^{2}$

\subsection{Case II: $\mathrm{H}_{0}=H_{0} \mathrm{e}_{2}$}

We have solved problem (8), (7) numerically.

The behaviour of $f, f^{\prime}, f^{\prime \prime}, g, g^{\prime}, g^{\prime \prime}$ is the same as in Case I. In particular, when $c<c_{r}$ the reverse flow appears.

We refer to Figures 2 and 3 for the solution of the problem.

The numerical integration furnishes $f^{\prime \prime}(0), g^{\prime \prime}(0), h, \alpha, \beta, \bar{\eta}_{f}$, and $\bar{\eta}_{g}$ : their values, when $M^{2}$ and $c$ change, are shown in Table 5.

We notice that if $M^{2}$ is fixed, then the descriptive quantities behave as in Case I when $c$ increases.

When $c$ is fixed, we find that if $M^{2}$ increases, then $f^{\prime \prime}(0), g^{\prime \prime}(0)$ increase, while the other parameters decrease.

Hence we have that the thickness $\delta$ of the boundary layer decreases when $M^{2}$ increases (as easily seen in Figures 6). In this case the thickness of the boundary layer is smaller than in Case I.

Table 6 shows that as the Hartmann number $M^{2}$ increases, then the value of $c_{r}$ for which the reverse flow does not occur decreases and when $M^{2}=0.7583$, 

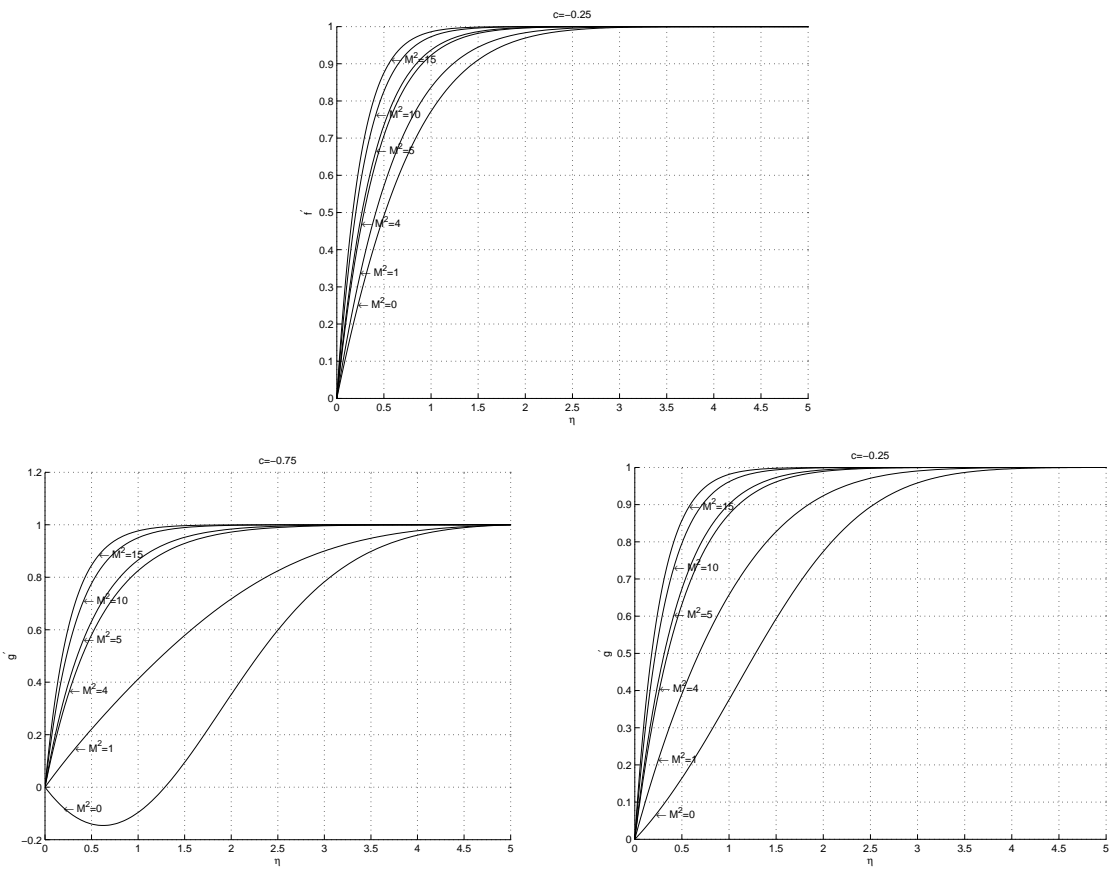

Figure 6: Case II: profiles of $f^{\prime}\left(6_{1}\right)$ and $g^{\prime}\left(6_{2,3}\right)$ for several values of $M^{2}$.

the reverse flow does not occur at all for any value of $c$. In this case

$$
\frac{\partial p}{\partial x_{3}}=-\rho a^{2} c x_{3}\left(c+2 M^{2}\right)
$$

from which one can see that the signs of $c$ and of $\left(c+2 M^{2}\right)$ modify the sign of $\frac{\partial p}{\partial x_{3}}$.

As far as the classification of the stagnation-point is concerned, we have found that $f^{\prime \prime}(0)+c g^{\prime \prime}(0)$ is always positive, so that the origin is a point of attachment. We remark that in this case $M^{2}$ directly influences $f$ and $g$, as we can see from system (8).

Moreover if $c>0$ or where there is the reverse flow, then the origin is a nodal point, while when $c<0$ and the reverse flow does not appear, it is a saddle point. These results are the same as for $M^{2}=0$. 


\begin{tabular}{cc}
\hline$M^{2}$ & $c_{r}$ \\
\hline 0.10 & -0.5024 \\
0.30 & -0.6479 \\
0.50 & -0.7922 \\
0.70 & -0.9342 \\
0.7583 & -0.9751 \\
0.7584 & no reverse flow \\
\hline
\end{tabular}

Table 6: Case II: Values $c_{r}$ when $M^{2}$ increases

\begin{tabular}{cccccccccc}
\hline$M^{2}$ & $c$ & $f^{\prime \prime}(0)$ & $g^{\prime \prime}(0)$ & $h$ & $\alpha$ & $\beta$ & $\bar{\eta}_{f}$ & $\bar{\eta}_{g}$ & $\delta$ \\
\hline 1 & -0.75 & 1.5941 & -0.4355 & -5.1702 & 0.5358 & 2.4378 & 2.1012 & 4.4881 & 4.4881 \\
1 & -0.25 & 1.5805 & 0.3035 & 0.2946 & 0.5472 & 1.3049 & 2.1626 & 3.5180 & 3.5180 \\
1 & 0.25 & 1.5950 & 0.8234 & 0.5869 & 0.5309 & 0.8112 & 2.0279 & 2.5816 & 2.5816 \\
1 & 1.00 & 1.6383 & 1.3216 & 0.5288 & 0.4950 & 0.5627 & 1.7971 & 1.9227 & 1.9227 \\
5 & -0.75 & 2.5541 & -0.3729 & -5.3339 & 0.3648 & 2.2644 & 1.5462 & 4.4113 & 4.4113 \\
5 & -0.25 & 2.5486 & 0.3663 & 0.0909 & 0.3676 & 1.1979 & 1.5692 & 3.3504 & 3.3504 \\
5 & 0.25 & 2.5547 & 0.8599 & 0.4457 & 0.3635 & 0.7748 & 1.5251 & 2.4881 & 2.4881 \\
5 & 1.00 & 2.5735 & 1.3434 & 0.4514 & 0.3530 & 0.5498 & 1.4309 & 1.8797 & 1.8797 \\
\hline
\end{tabular}

Table 7: Case III: descriptive quantities of motion for some values of $c$ and $M^{2}$

\subsection{Case III: $\mathrm{H}_{0}=H_{0} \mathrm{e}_{3}$}

We have solved problem (9), (7) numerically.

The behaviour of $f, f^{\prime}, f^{\prime \prime}, g, g^{\prime}, g^{\prime \prime}$ is the same as in Case I and Case II. In particular, when $c<c_{r}$ the reverse flow appears.

We refer to Figures 2 and 3 for the solution of the problem.

The numerical integration furnishes $f^{\prime \prime}(0), g^{\prime \prime}(0), h, \alpha, \beta, \bar{\eta}_{f}$, and $\bar{\eta}_{g}$ : their values, when $M^{2}$ and $c$ change, are shown in Table 7.

We notice that if $M^{2}$ is fixed, then the descriptive quantities behave as in Case I when $c$ increases, even if they do not change in a relevant way compared to the previous cases.

When $c$ is fixed, we note that if $M^{2}$ increases, then $f^{\prime \prime}(0), g^{\prime \prime}(0)$ increase, while the other parameters decrease.

Hence the thickness $\delta$ of the boundary layer decreases when $M^{2}$ increases (as easily seen in Figures 7) and the boundary layer is thinner than in Case II.

Table 8 shows that as the Hartmann number $M^{2}$ increases, then the value of $c_{r}$ for which the reverse flow does not occur (i.e. when $g^{\prime \prime}(0)=0$ ) decreases very slowly, so that in this case the influence of the magnetic field is much less 

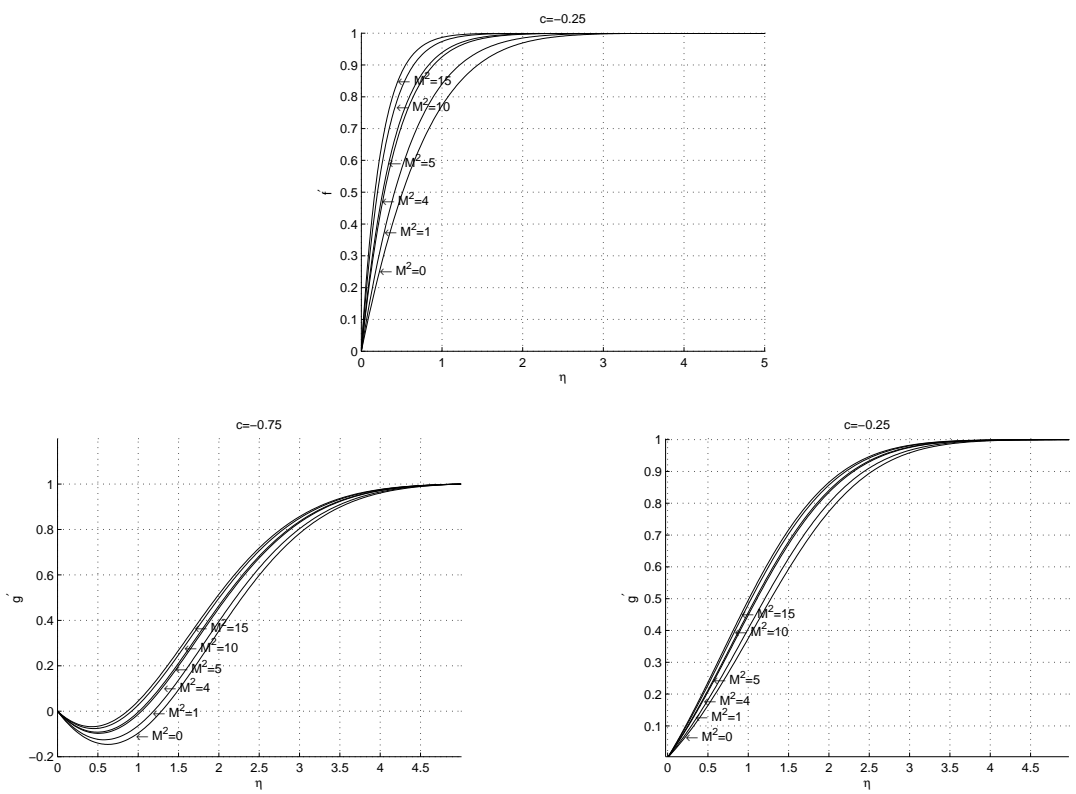

Figure 7: Case III: profiles of $f^{\prime}\left(7_{1}\right)$ and $g^{\prime}\left(7_{2,3}\right)$ for several values of $M^{2}$.

\begin{tabular}{cc}
\hline$M^{2}$ & $c_{r}$ \\
\hline 1.00 & -0.4564 \\
10.00 & -0.5295 \\
50.00 & -0.5768 \\
100.00 & -0.5904 \\
\hline
\end{tabular}

Table 8: Case III: Values $c_{r}$ when $M^{2}$ increases

significant with respect to CaseS I-II. In this regard we observe that $\frac{\partial p}{\partial x_{3}}=$ $-\rho a^{2} c^{2} x_{3}, \frac{\partial p}{\partial x_{1}}=-\rho a^{2} x_{1}\left(1+M^{2}\right)$ so that they have the same sign that they would in the absence of the external magnetic field. In particular, we see that the reverse flow always appears for physically meaningful values of $M^{2}$.

As in the previous case, the origin is always a point of attachment. As one can see, $M^{2}$ directly influences $f$ and only indirectly influences $g$ in system (9), so that when $M^{2}$ increases, $f^{\prime \prime}(0)$ becomes much greater than $g^{\prime \prime}(0)$.

Moreover if $c>0$ or where there is the reverse flow, the origin is a nodal point, while when $c<0$ and the reverse flow does not appear, it is a saddle 
point.

\section{Conclusions}

A numerical study is performed for the steady three-dimensional MHD stagnationpoint flow of an incompressible, homogeneous electrically conducting Newtonian fluid over a flat plate in the presence of a uniform external magnetic field. This paper completes our previous analysis concerning the Newtonian fluids ([4]) where we have proved that, if we impress an external magnetic field $\mathbf{H}_{0}$, and we neglect the induced magnetic field, then the steady three-dimensional MHD stagnation-point flow is possible if, and only if, $\mathbf{H}_{0}$ has the direction of one of the coordinate axes.

We have solved numerically the three non-linear ordinary boundary value problems by using the MATLAB routine bvp4c.

The results of Cases I-III are totally new.

The following conclusions can be made for the three cases considered.

- The presence of the magnetic field modifies the thickness $\delta$ of the boundary layer which decreases as $M^{2}$ (the Hartmann number) increases.

- $\mathbf{H}_{0}$ tends to prevent the occurrence of the reverse flow in a relevant way in Cases I-II.

- The three-dimensional displacement thickness $h$ can be positive or negative. When the reverse flow appears it is negative.

- In Case II, $\delta$ and the value of $M^{2}$ starting from which the reverse flow does not appear for any values of $c$ are smaller than in the other cases.

- The stagnation-point has been classified as nodal or saddle point and as attachment or separation point. In Case II and Case III, as for $M^{2}=0$, the origin is a point of attachment.

In Case I, we find a new result: when $M^{2}$ is sufficiently large and $c$ assumes suitable negative values, then the stagnation-point becomes a separation point.

Further in all cases if $c>0$ or where there is the reverse flow, the origin is a nodal point, while when $c<0$ and the reverse flow does not appear, it is a saddle point. 


\section{References}

[1] F. Homman, Der Einfluss grosser Zahigkeit bei der Stromung um den Zylinder und um die Kugel, Zeitschrift fur Angewandte Mathematik und Mechanik, 16 (1936), 153-164.

[2] L. Howarth, The boundary layer in three dimensional flow - Part II: The flow near a stagnation point, Philosophical Magazine Series 7, 42 (1951), 1433-440.

[3] A. Davey, Boundary layer flow at a saddle point of attachment, Journal of Fluid Mechanics, 10 (1961), 593-610.

[4] A. Borrelli, G. Giantesio, M.C. Patria, Three-dimensional MHD stagnation point-flow of a Newtonian and a micropolar fluid, International Journal of Pure and Applied Mathematics, 73 (2011), 165-188.

[5] S.P. Hastings, An existence theorem for a problem from boundary layer theory, Archive of Rational Mechanics and Analysis, 33 (1969), 103-109.

[6] S.P. Hastings, Existence for a Falkner-Skan type boundary value problem, Journal of Mathematical Analysis and Applications, 31 (1970), 15-23.

[7] S. Bhattacharyya, A.S. Gupta, MHD flow and heat transfer at a general three-dimensional stagnation point, International Journal of Non-Linear Mechanics, 33 (1998), 125-134.

[8] P. Drazin, N. Riley, The Navier-Stokes equations. A Classification of Flows and Exact Solutions, London Mathematical Society, Lecture Notes Series 334, Cambridge University Press (2007).

[9] A. Davey, Rotational flow near a forward stagnation point, The Quarterly Journal of Mechanics and Applied Mathematics, 26 (1963), 33-59.

[10] M.J. Lighthill, Laminar Boundary Layers (Ed. L. Rosenhead), Dover Publ., Oxford (1963).

[11] L.F. Shampine, I. Gladwell, S. Thompson, Solving ODEs with MATLAB, Cambridge University Press (2003). 